\title{
Aktualisieren Sie Ihre Fachkunde im Strahlenschutz von zu Hause aus
}

Die Akademie Online der DRG bietet erstmals Kurse zur Aktualisierung der Fachkunde an. Die Buchung der Module B (Strahlenschutzverordnung) und C (Röntgenverordnung) steht ab sofort online zur Verfügung. Das Modul A (Strahlenschutzrecht) inklusive Prüfung bleibt eine Präsenzveranstaltung, die wir im Mai auf dem Röntgenkongress und der Jahrestagung der Deutschen Gesellschaft für HNO anbieten.

Bitte beachten Sie, dass Sie Modul A in jedem Fall belegen müssen. Zusätzlich benötigen Sie Modul C (Röntgen) und/oder B (Strahlentherapie und Nuklearmedizin).
Termine für die 3-stündige Online-Fortbildung finden Sie unten. Für die Teilnahme gelten pro Modul folgende Preise:

\section{DRG-Mitglied: 30,00 Euro}

VMTB-Mitglied: 25,00 Euro

DRG/VMTB-Nicht-Mitglied: 50,00 Euro

Zur Teilnahme benötigen Sie einen internetfähigen Computer mit Tonausgabe. Nach der Registrierung werden Ihnen die Zugangsdaten zugesendet. Auf unserer Homepage www.drg.de können Sie sich ab sofort für die ersten Kursangebote anmelden:
Röntgendiagnostik (RöV)

Montag, 15. April 2013

(Modul C, nach RöV)

oder

Donnerstag, 2. Mai 2013

(Modul C, nach RöV)

Nuklearmedizin und Strahlentherapie (StrlSchV)

Freitag, 3. Mai 2013

(Modul B, nach StrlSchV)

\section{Strahlenschutzrecht und Prüfung} (muss auf jeden Fall belegt werden) Samstag 1. Juni 2013 (Modul A), Dt. Röntgenkongress (DRK), Hamburg oder

Sonntag 12. Mai 2003 (HNO-Kongress, Nürnberg)

Informationen zur Zertifizierung der Kurse durch die Ärztekammer stehen in Kürze auf www.drg.de zur Verfügung. 